\title{
Quantum coherent control of $\pi$-electron rotations in a nonplanar Chiral molecule
}

\author{
Hirobumi Mineo ${ }^{1, a}$, Masahiro Yamaki $^{\text {b }}$, Yoshiyuki Teranishic ${ }^{\mathrm{c}}$, Hiroki Nakamura ${ }^{\mathrm{b}}$, \\ Sheng Hsien Lin ${ }^{b}$, Yuichi Fujimurab
}

${ }^{a}$ Institute of Atomic and Molecular Sciences, Academia Sinica, No.1, Sec. 4,Roosevelt Rd., Taipei 106 Taiwan.E-mail: mineo@gate.sinica.edu.tw

${ }^{b}$ Department of Applied Chemistry, Institute of Molecular Science, National Chiao-Tung University, 1001 University Road, Hsin-Chu 300 Taiwan. E-mail: fujimurayuichi@m.tohoku.ac.jp

${ }^{c}$ Institute of Physics, National Chiao-Tung University,1001 University Road., Hsin-Chu 300, Taiwan. Email: tera@mail.nctu.edu.tw

\begin{abstract}
The results of a theoretical investigation of coherent $\pi$-electron dynamics for nonplanar $(P)$ 2,2'-biphenol induced by ultrashort linearly polarized UV pulses are presented. The coherent ring currents originate from an excitation of a pair of quasi-degenerate electronic states by an ultrashort linearly polarized UV laser pulse. The magnitudes of the electronic ring currents are expressed as the sum of expectation values of the corresponding operators in the two phenol rings ( $L$ and $R$ rings). Here, $L(R)$ denotes the phenol ring in the left (right)-hand side of $(P)$ 2,2'-biphenol. For $\pi$ electrons in (P)-2,2'-biphenol, there are four possible rotational patterns. The bond current of the bridge bond linking the L and R rings is zero for the symmetric coherent state, while it is nonzero for the antisymmetric coherent state.
\end{abstract}

2013 International Workshop on Computational Science and Engineering (IWCSE 2013), October 14-17, 2013, Taipei, Taiwan

${ }^{1}$ Speaker 


\section{Introduction}

Recently there have been wide interest in ultrafast coherent dynamics of electronic dynamics in molecules ${ }^{1-4}$. Especially $\pi$ electrons in aromatic rings are considered to play an important role for organic electronics. There have been several theoretical works of the generation and control of $\pi$ electron motions in molecular sysetms by using ultrafast UV laser pulse $^{5-16}$. The $\pi$-electron ring current in Mg-porphyrin are induced by a few cycle of circularly polarized UV laser pulse ${ }^{5-7}$. Here photon angular momentum is transfered to degenerated electronic excited states by circularly polarized laser pulse. The lineraly polarized (LP) UV laser pulse is used to create $\pi$-electron ring current in 2,5 -dichloro[n] $(3,6)$ pyrazinophane, which is a planar chiral molecule and has no degenerate electronic excited states ${ }^{9-12}$. That is that the angular momentum or coherent ring current are created through a coherent excitation of a pair of quasi-degenerate electronic excited states.

In this work we use $(P)-2,2^{2}$-biphenol, which is a typical nonplanar chiral aromatic molecule of axial chirality (see Fig.1). Figure 2 shows that there are four possible rotational paterns (CC, AA, CA and AC) where $\mathrm{C}$ and A refer to clockwise and anticlockwise rotations respectively, and the first and second letters in each patterns refer to left $(L)$ and right $(R)$ phenol rings respectively. The right hand side of figure 2 represents the initial rotational direction of $\pi$ electrons in the case in which a super position of two electronic excited states a and b1, with different irreducible representation of point group $\mathrm{C}_{2}$ is prepared. $\mathbf{e}_{a b 1}^{(+)}\left(\mathbf{e}_{a b 1}^{(-)}\right)$represents preparation of the relative phase between a and $\mathrm{b} 1$ is in-phase (out-phase). Based on these rotational patterns we clarify how the ring currents and bridge current are created, and show time evolution behaviors of them ${ }^{13-16}$.

In section 2 we show expressions of the ring current and bond bridge current in aromatic molecules. In section 3 we perform the numerical simulation of the ring current and bond bridge current in order to discuss a transfer of the coherent ring currents through a bridge bond. In section 4 the summary and conclusion of this paper are given.

\section{Coherent ring current}

Expectation values of the electronic current operators of an aromatic molecule excited by a pulse laser are generally expressed as

$$
\langle\hat{O}(\vec{r}, t)\rangle=n \int d^{3} r_{1} \cdots d^{3} r_{n} \delta\left(\vec{r}-\vec{r}_{1}\right) \Psi^{*}(t) \hat{O}\left(\vec{r}_{1}\right) \Psi(t) .
$$

Here, $\hat{O}\left(\vec{r}_{1}\right)$ is a single-electron operator for the current, $\Psi(t)$ is an electronic wave function at time $t, n$ is the number of electrons, and $\vec{r}_{i}$ denotes the electron coordinates of the $i$-th electron. Since we are interested in optically allowed electronic excited states of conjugated aromatic molecules, we expand the electronic wave function in terms of electronic 
configurations within CIS calculations, the ground electronic $\Phi_{0}$ and singly excited electronic ones $\Phi_{\alpha}$ as

$$
\Psi(t)=C_{0}(t) \Phi_{0}+\sum_{\alpha} C_{\alpha}(t) \Phi_{\alpha},
$$

where $\Phi_{0}$ is given as $\Phi_{0}\left(\vec{r}_{1}, \cdots, \vec{r}_{n}\right)=\left\|\phi_{1} \cdots \phi_{a} \cdots \phi_{b} \cdots \phi_{n}\right\|$ with $\phi_{n} \equiv \phi_{n}\left(\vec{r}_{n}\right)$. Here, $\phi_{a}$ and $\phi_{b}$ are among the $n$ occupied orbitals, and $\Phi_{\alpha}$ is the electronic wave function for a singly excited electronic configuration $\alpha: a \rightarrow a^{\prime}$, i.e., single electron transition from occupied MO $a$ to unoccupied MO $a^{\prime}, \Phi_{\alpha}\left(\vec{r}_{1}, \cdots, \vec{r}_{n}\right)=\left\|\phi_{1} \cdots \phi_{a^{\prime}} \cdots \phi_{n}\right\|$. A $\pi$ orbital $\phi_{k}$ associated with optical transition is expanded in terms of a linear combination of atomic orbitals $\chi_{i}$ as

$$
\phi_{k}=\sum_{i} c_{k, i} \chi_{i},\left(k=a, a^{\prime}, b, b^{\prime}\right),
$$

where $i$ specifies the atomic orbital and $c_{k, i}$ is the molecular orbital coefficient.

An electric current passing through a surface $S$ at time $t$ is generally defined as

$$
\langle J(t)\rangle \equiv \int_{S} d^{2} r_{\perp} \vec{n}_{\perp} \cdot\langle\hat{J}(\vec{r}, t)\rangle .
$$

Here, $\vec{n}_{\perp}$ is a unit vector perpendicular to a surface $S$, and $\langle\hat{J}(\vec{r}, t)\rangle \equiv\langle\Psi(t)|\hat{J}(\vec{r})| \Psi(t)\rangle$. This can be expressed as

$$
\langle\hat{J}(\vec{r}, t)\rangle=\frac{2 n e \hbar}{m_{e}} \sum_{\alpha<\beta} \operatorname{Im}\left(C_{\beta}(t) C_{\alpha}^{*}(t)\right) \sum_{i j}\left(\delta_{a b} C_{a^{\prime} i}^{*} c_{b^{\prime} j}+\delta_{a^{\prime} b}, C_{a i}^{*} c_{b j}\right) \chi_{i}^{*} \vec{\nabla} \chi_{j},
$$

where $\hat{J}(\vec{r})=\frac{e \hbar}{2 m_{e} i}(\vec{\nabla}-\overleftarrow{\nabla})$ is the current density operator. Here, $\vec{\nabla}(\stackrel{\leftarrow}{\nabla})$ denotes the nabra operating the atomic orbital on the right-hand (left-hand) side. Surface integration in Eq. (4) is carried out over a half-plane $S$.

Since the $L$ and $R$ rings are not round but consist of nonequivalent $\mathrm{C}-\mathrm{C}$ bonds, we introduce the bond current $\langle J(t)\rangle_{i j}$ from the nearest neighbor atoms at sites $j$ to $i$, which is given in terms of inter-atomic bond current (IABC) $J_{i j}^{I A B C}$ and Eq. (4) as

$$
\langle J(t)\rangle_{i j}=\frac{2 n e \hbar}{m_{e}} \sum_{\alpha<\beta} \operatorname{Im}\left(C_{\beta}(t) C_{\alpha}^{*}(t)\right)\left(\delta_{a b}\left(c_{a^{\prime} i}^{*} c_{b^{\prime} j}-c_{b^{\prime} i}^{*} c_{a^{\prime} j}\right)+\delta_{a^{\prime} b^{\prime}}\left(c_{a i}^{*} c_{b j}-c_{b i}^{*} c_{a j}\right)\right) J_{i j}^{I A B C},
$$

where

$$
J_{i j}^{I A B C}=\int_{S} d^{2} r_{\perp} \chi_{i}^{*} \vec{n}_{\perp} \cdot \vec{\nabla} \chi_{j}\left(\equiv J_{i j}^{I A B C(S \text { at center })}\right) .
$$

Here, surface $S$ is set to be perpendicular to the $C_{i}$ and $C_{j}$ bond at the center, and 
$\vec{n}_{\perp}=\frac{\vec{r}_{i}-\vec{r}_{j}}{\left|\vec{r}_{i}-\vec{r}_{j}\right|} \cdot c_{k, l} \quad\left(\left(k=a, b, a^{\prime}, b^{\prime}, l=i, j\right)\right)$ is the molecular orbital coefficient with occupied orbital $a, b$ or unoccupied orbital $a^{\prime}, b^{\prime}$ and atomic cite $l . \quad \chi_{l}$ is the atomic orbital of the cite l. It should be noted that the laser intensity dependence is involved in the imaginary part of the off-diagonal density matrices, $\operatorname{Im}\left(C_{\beta}(t) C_{\alpha}^{*}(t)\right)$. We now define an effective ring current $J_{K}$ along ring $K=L, R$ by taking the average over the all bond currents as $J_{K} \approx \frac{1}{N_{K}} \sum_{(i j) \subset K}^{N_{K}}\langle J(t)\rangle_{i j}$, where $N_{\mathrm{K}}$ is the number of bonds of ring $K$.

\section{Numerical results and discussions}

The geometry of 2,2'-biphenol in the ground state was optimized by using the DFT B3LYP level of theory in the GAUSSIAN09 program $^{17}$. The energies of the electronic excited states were calculated at the optimized ground state geometry at the TDDFT B3LYP level of theory. The $6-31 \mathrm{G}+(\mathrm{d}, \mathrm{p})$ basis sets were used throughout our calculations. The dihedral angle between the two phenyl groups is found to be 108.8 degrees from our DFT calculations. For generation of coherent angular momentum and ring current of $(P)-2,2$ '-biphenol, we focus on the three optically allowed excited states $\left(a, b_{1}\right.$ and $\left.b_{2}\right)$ whose energies range from 6.67 to 6.84 $\mathrm{eV}$ as shown in Fig. 1. The transition energies to the $a, b_{1}$ and $b_{2}$ states, which were calculated at the optimized ground state geometry using the TD-DFT B3LYP level of theory are 6.67, 6.78, and $6.84 \mathrm{eV}$, respectively. The transition dipole moment from the ground state to excited state $a$ (A), $\boldsymbol{\mu}_{g a}(=(0,0,-0.77))$, is parallel to the Z-axis, and the others, $\boldsymbol{\mu}_{g b 1}(=(0.08,1.93,0))$ and $\boldsymbol{\mu}_{g b 2}(=(1.24,-0.34,0))$, are nearly orthogonal to each other in the $X Y$-plane as shown in Fig. 1

In figure 3(a) directions of the ring current $J_{L}\left(J_{R}\right)$ on $L(R)$ phenol ring, and the bridge bond current are defined. In figure 3(b) we shine the $\pi$-pulse gaussian-shaped LP laser pulse with the amplitude $1.2 \mathrm{GV} / \mathrm{m}$ and pulse duration $64 \mathrm{fs}$. Here polarization direction of the laser pulse is chosen as in-phase direction to coherently excite to quasi-degenerate electronic excited states $b_{1}$ and $b_{2}$. It is observed that ring currents $J_{L}$ and $J_{R}$ oscillate in the same phase, whereas the bridge bond current $J_{B}$ is permanently zero. On the other hand, in figure 3(c) we shine the $\pi$ pulse gaussian-shaped LP laser pulse with the amplitude $2.5 \mathrm{GV} / \mathrm{m}$ and pulse duration $38 \mathrm{fs}$. Similarly to Fig. 3(b), the polarization direction is in-phase direction to excite to quasidegenerate electronic excited states $a$ and $b_{1} . J_{L}$ and $J_{R}$ oscillate in the opposite phase, and as a characteristic feature $J_{B}$ is generated, and the amplitude of transferred current is smaller than the amplitudes of ring currents. Transfer of ring currents will occur if the created coherent excited stated states belong to different irreducible representations A and B. 


\section{Summary and conclusion}

In this paper, we presented the results of a theoretical study on coherent quantum dynamics of $\pi$ electrons in (P)-2,2'-biphenol. (P)-2,2'-biphenol is a typical nonplanar chiral aromatic molecule with axial chirality, and a pair of quasi-degenerate excited states in the two phenol rings is coherently excited by an ultrashort linearly polarized UV pulse. This results in the generation of coherent two-dimensional angular momentum and the resultant ring current on two different molecular planes. An expression for the coherent $\pi$-electron ring current is derived in a closed form. The bond current is given as the electric current through a surface perpendicular to the bond at the center, and the expression for bond current is derived within an LCAO MO approximation. Results of simulations of electric ring current quantum beats are presented to demonstrate the time-dependent behaviors of two coherent excited states, $\left(b_{1}+b_{2}\right)$ with A symmetry, $\left(a+b_{1}\right)$ with B symmetry in the point group $\mathrm{C}_{2}$. It was found that the value of the bridge bond current depends on the symmetry of the electronic coherence, i.e., the value vanishes for the electronic coherent state $\left(b_{1}+b_{2}\right)$ with A symmetry, while there are nonzero for the two electronic coherence states $\left(a+b_{1}\right)$ with B symmetry. The initial direction of the coherent ring current for each electronic coherence state $\left(a+b_{1}\right)$ with B symmetry can be determined by evaluating the corresponding bridge bond current density.

It should be noted that there is a difference between the mechanism of a unidirectional $\pi$ electron ring current in aromatic molecules with degenerated electronic states, $\mathrm{Mg}$-porphyrin and benzene, and that of of coherent $\pi$-electron ring current in a non-degenerate aromatic molecule, $(P)-2,2$ '-biphenol. For the former molecules, population transfer from the ground to a degenerated electronic excited state by a circularly polarized UV laser pulse is the mechanism, while for the latter case, creation of a coherent electronic state from two quasi-degenerate electronic excited states is the mechanism. 


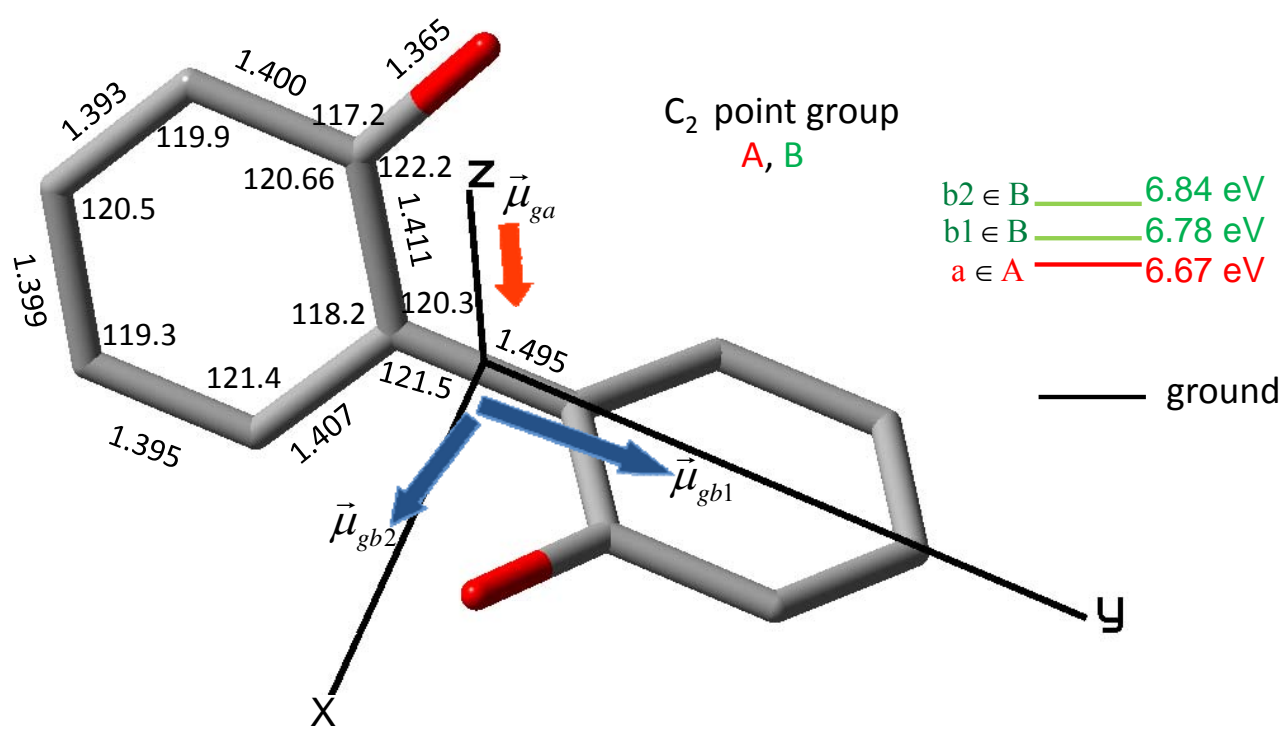

Fig. 1. Geometrical structure of (P)-2,2'-biphenol and transition dipole moments with three electronic excited states $a, b 1$ and $b 2$.

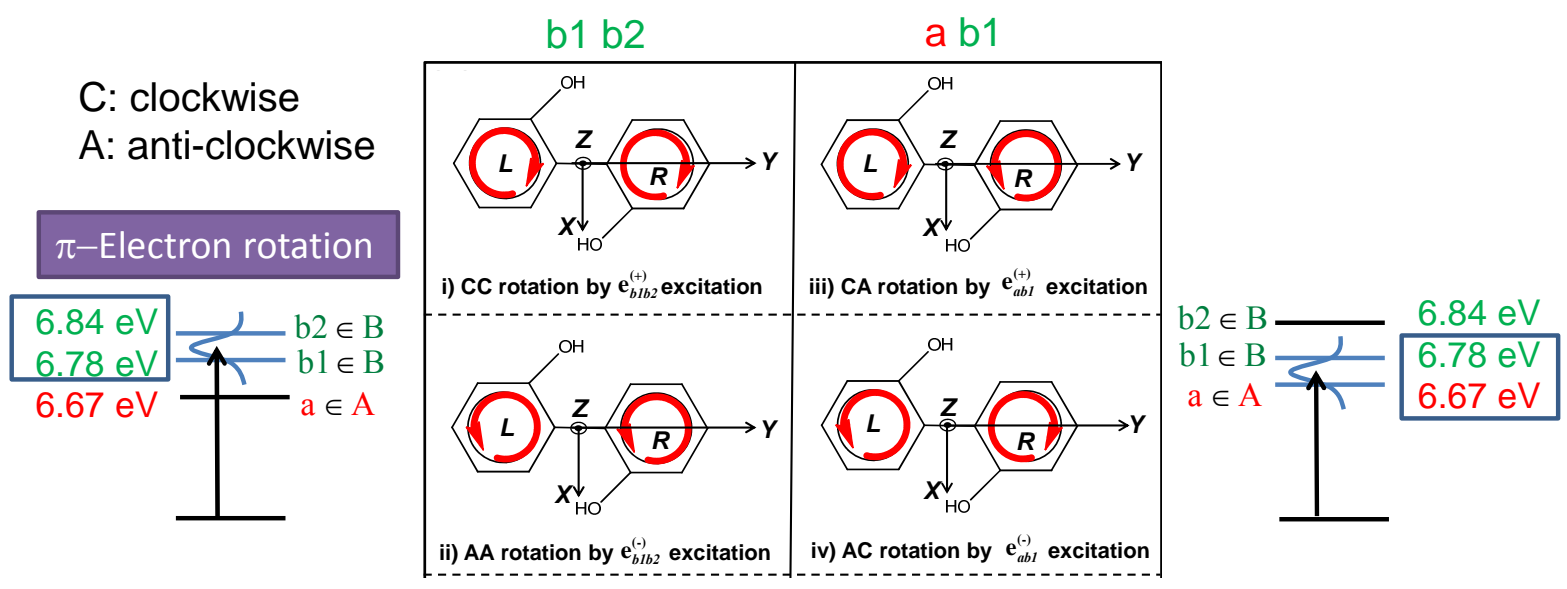

Fig. 2. Four patterns of ring current rotations induced by coherent excited states $\left(b_{1} \pm b_{2}\right)$ and $\left(a \pm b_{1}\right)$. Here $+(-)$ represents a polarization direction of laser pulse which creates a combination of two coherent excited states with in-phase (out-phase). 
(a)

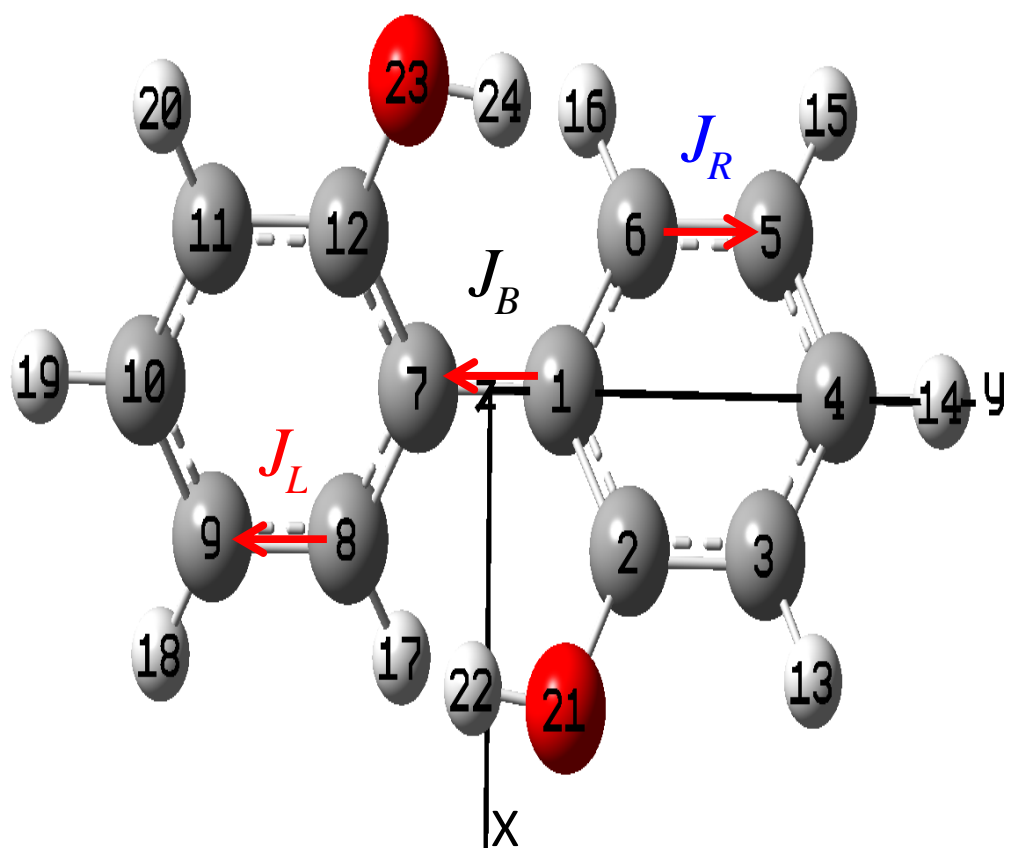

(b)

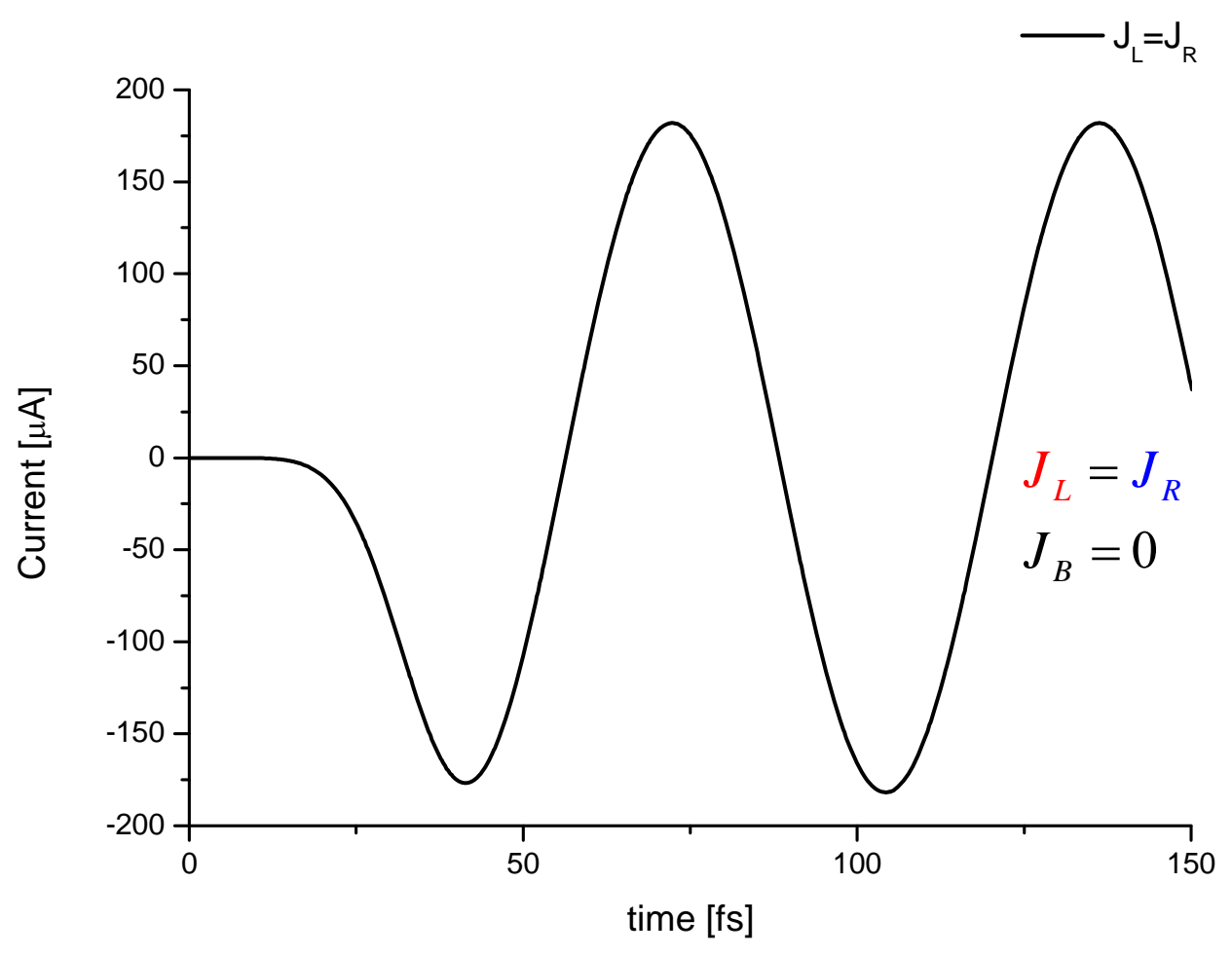

(c) 


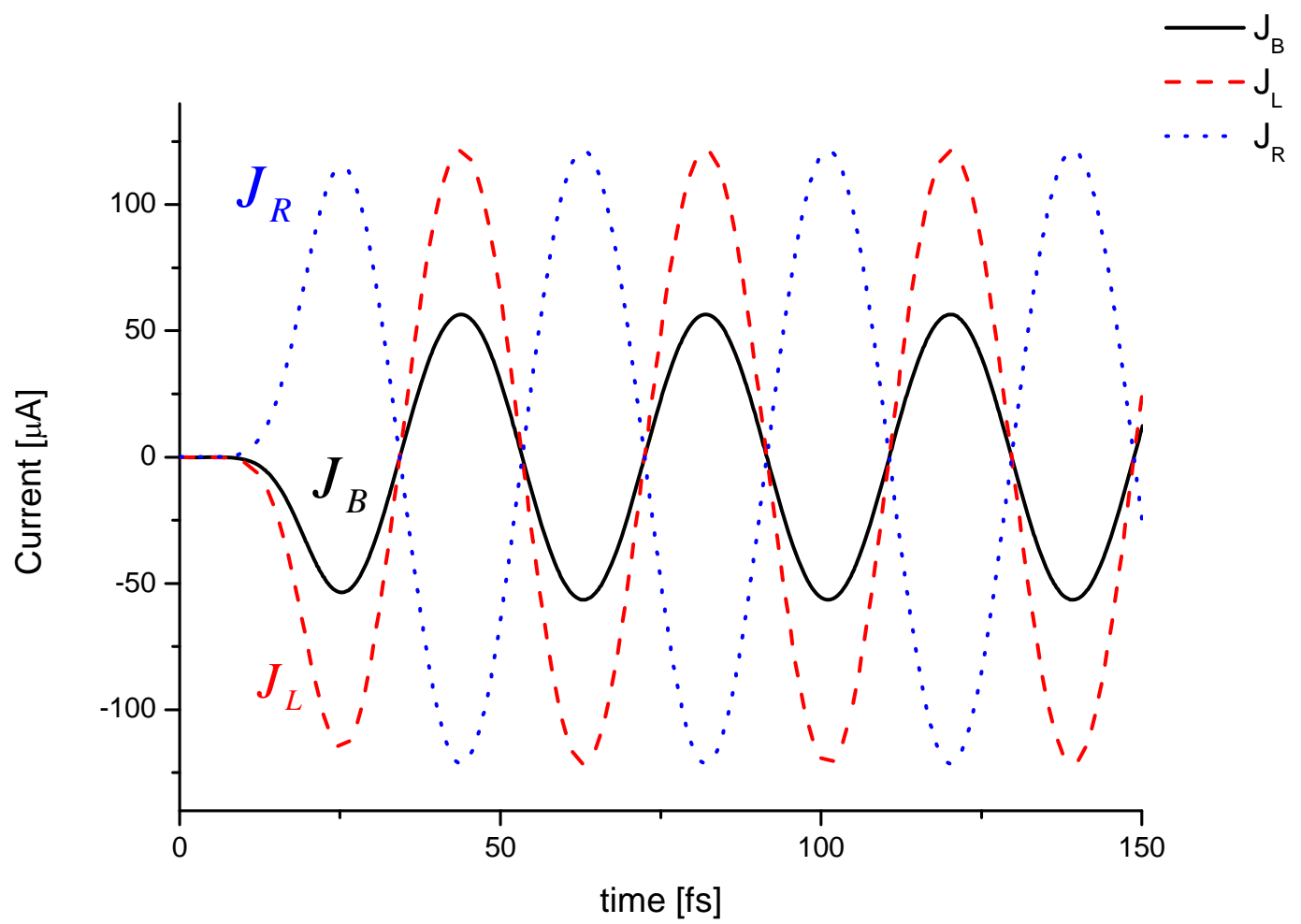

Fig. 3. (a) Definitions of directions of the ring current $J_{L}\left(J_{R}\right)$ on $L(R)$ phenol ring, and the bridge bond current $J_{B}$ are given. (b) Time evelution of ring currents $J_{L}, J_{R}$ and bridge bond current $J_{B}$ are plotted. Here coherent electronic excited states $b_{1}$ and $b_{2}$ are created with in-phase relative coherence by a LP laser pulse with the amplitude $1.2 \mathrm{GV} / \mathrm{m}$ and pulse duration $64 \mathrm{fs}$. (c) Time evelution of ring currents $J_{L}, J_{R}$ and bridge bond current $J_{B}$ are plotted. Here coherent electronic excited states a and $b_{1}$ are created with in-phase relative coherence by a LP laser pulse with the amplitude $2.5 \mathrm{GV} / \mathrm{m}$ and pulse duration $38 \mathrm{fs}$.

\section{References}

[1] P. Tzallas, E. Skantzakis, J. E. Kruse, and D. Charalambidis, Progress in Ultrafast Intense Laser Science (Springer, Heidelberg) 7, 163 (2011).

[2] Y. Fujimura, H. Sakai, Electronic and Nuclear Dynamics in Molecular Systems, World Scientific, Singapore, p. 117 (2011).

[3] Y. Nabekawa and K. Midorikawa, Adv. in Multiphoton Processes and Spectroscopy (World Scientific Singapore), V01. 18, 1 (2008).

[4] M. Lein and C. C. Chirilă, Adv. in Multiphoton Processes and Spectroscopy (World Scientific Singapore), V01. 18, 69 (2008).

[5] I. Barth and J. Manz, Angew. Chem. Int. Ed. 45, 2962 (2006). 
[6] I. Barth, J. Manz, Y. Shigeta, and K. Yagi, J. Am. Chem. Soc. 128, 7043 (2006).

[7] I. Barth and J. Manz, Progress in Ultrafast Intense Laser Science VI, Springer 2010, 21 (2010).

[8] K. Nobusada and K. Yabana, Phys. Rev. A 75, 032518 (2007).

[9] M. Kanno, H. Kono, and Y. Fujimura, Angew. Chem. Int. Ed. 45, 7995 (2006).

[10] M. Kanno, K. Hoki, H. Kono, Y. Fujimura, J. Chem. Phys. 127, 204314 (2007).

[11] M. Kanno, H. Kono, Y. Fujimura, S. H. Lin, Phys. Rev. Lett. 104, 108302 (2010).

[12] M. Kanno, H. Kono, and Y. Fujimura, Progress in Ultrafast Intense Laser Science VII, Springer, 53 (2011).

[13] H. Mineo, M. Yamaki, Y. Teranish, M. Hayashi, S. H. Lin, and Y. Fujimura, J. Am. Chem. Soc. 134, 14279 (2012).

[14] H. Mineo, M. Kanno, H. Kono, S. D. Chao, S. H. Lin, and Y. Fujimura, Chem. Phys. 392, 136 (2012).

[15] H. Mineo, S.H. Lin, and Y. Fujimura, J. Chem. Phys. 138, 074304 (2013).

[16] H. Mineo, S.H. Lin, Y. Fujimura, J. Xu, R.X. Xu, and Y.J. Yan, J. Chem. Phys. 139, 214306 (2013).

[17]Gaussian 09, Revision E.01, M. J. Frisch, et al., Gaussian, Inc., Wallingford CT, 2009. 